\title{
Determination of neutron energy spectra for lunar meteorites studied from REE isotopic compositions
} \author{
H. HIDAKA \\ ${ }^{1}$ Department of Earth and Planetary Sciences, Nagoya \\ University, Nagoya 464-8601, Japan (*correspondence: \\ hidaka@eps.nagoya-u.ac.jp) \\ ${ }^{2}$ Space Science Laboratory, University of California, \\ Berkeley, CA 94720-7450, USA \\ ${ }^{3}$ Department of Physics and Astronomy, Purdue University, \\ West Lafayette, IN 47907-2036, USA \\ ${ }^{4}$ Department of Science and Engineering, National Museum \\ of Nature and Science, Tsukuba 305-0005, Japan
}

It is known that most lunar meteorites have complicated cosmic-ray exposure (CRE) histories with relatively longer CRE ages than the other stony meteorites, and that their transfer durations from Moon to Earth are quite short [1]. Our major interest is the CRE circumstances of individual lunar meteorites on the Moon.

Neutrons arising at the surface of the Moon are produced from the interaction of cosmic rays with the lunar surficial materials by spallation reactions. Since the thermalization degree of the neutrons depends mainly upon the depths and the chemical compositions of the materials [2], the fine structure of the neutron energy spectrum may provide some insights into the environmental situations around the location of individual meteorites on the Moon. In this study, the neutron energy spectrum in the range of thermal and epithermal regions at the lunar surface was investigated from the combination of the isotopic variations of $\mathrm{Sm}$ and $\mathrm{Gd}$ by the thermal neutron capture reactions, and of $\mathrm{Er}$ and $\mathrm{Yb}$ by the epi-thermal neutron captuer reactions.

Eleven lunar meteorites, NWA 479, NWA 482, NWA 2995, NWA 2996, NWA 3163, NWA 4734, NWA 4932, NWA 5000, Dhofar 081, Dhofar 910, Dhofar 911, whose spallogenic products such as ${ }^{10} \mathrm{Be},{ }^{26} \mathrm{Al},{ }^{36} \mathrm{Cl}$ and ${ }^{41} \mathrm{Ca}$ were already reported [e.g., 1], were used in this study. For further study to obtain new information on the neuron energy induced by CRE, systematic isotopic analyses of $\mathrm{Sm}, \mathrm{Gd}, \mathrm{Er}$ and $\mathrm{Yb}$ were performed on the eleven samples.

The burial depths on the Moon, CRE duration, neutron fluences, and neutron energy spectra for the eleven samples were estimated from the data set given by spallogenic and neutron-captured products.

[1] Nishiizumi et al. (1996) MAPS, 31, 893-896. [2] Spergel et al. (1986) JGR, 91, D483-D494. 